Chirurg 2016 $\cdot 87: 72$

DOI 10.1007/s00104-015-0130-9

Online publiziert: 17. Dezember 2015

c) Springer-Verlag Berlin Heidelberg 2015

CrossMark

J. Reibetanz • C.T. Germer

Klinik für Allgemein-, Viszeral-, Gefäß- und Kinderchirurgie, Universitätsklinik Würzburg, Würzburg, Deutschland

\title{
Pathologisches Outcome nach laparoskopischer und offener Rektumkarzinomchirurgie
}

\section{Die ALaCaRT-Multicenterstudie}

\section{Ergebnisse}

Im Zeitraum zwischen 2010 und 2014 konnten aus 24 Zentren in Australien und Neuseeland insgesamt 475 Patienten randomisiert werden (238 laparoskopisch, 237 offen). Beide Kollektive waren vergleichbar hinsichtlich Alter, BodyMass-Index, Komorbidität und klinischem Tumorstaging. Jeweils die Hälfte der Patienten hatte eine neoadjuvante Radiochemotherapie erhalten, $35 \%$ der Tumoren je Gruppe lagen im unteren Rektumdrittel. Eine erfolgreiche Resektion konnte bei 194 Patienten (82\%) der laparoskopisch vs. 208 Patienten (89\%) der offen operierten Patienten erreicht werden („risk difference" $-7 \%$ [95\%-Konfidenzinter$\operatorname{vall(KI)~}-12,4$ bis $\infty$ ], $p=0,38$ für Nichtunterlegenheit). Ein negativer CRM fand sich bei 222 Patienten (93\%) der laparoskopischen Gruppe vs. 228 Patienten (97\%) der offen operierten Patienten („risk difference“ $-3,7 \%$ ), ein tumorfreier aboraler Resektionsrand bei 236 Patienten (99\%) der laparoskopischen und bei 234 Patienten (99\%) der offenen Gruppe (,risk difference“-0,4\%). Eine komplette mesorektale Exzision konnte bei 206 Patienten $(87 \%)$ der laparoskopisch operierten Patienten und 216 Patienten (92\%) der offen operierten Patienten erreicht werden. Die Konversionsrate betrug 9\%. Unterschiede in der Dauer des stationären Aufenthaltes, der Aufenthaltsdauer auf der Intensivstation oder hinsichtlich der MajorMorbidität ließen sich nicht nachweisen.

\section{Diskussion und Fazit des Reviewers}

Die ALaCaRT-Studie konnte insgesamt die Nichtunterlegenheit der laparo- skopischen gegenüber der offenen Rektumkarzinomchirurgie hinsichtlich der gewählten pathologischen Endpunkte nicht nachweisen. Die Ergebnisse widersprechen damit kürzlich publizierten Multicenterstudien [2] und mahnen zugleich zur Vorsicht bei der Anwendung der laparoskopischen Rektumkarzinomchirurgie. Nichtsdestotrotz, entscheidende Kriterien für die Anwendbarkeit der Laparoskopie beim Rektumkarzinom sollten nicht allein auf den hier präsentierten chirurgisch-pathologischen Kriterien (z. B. CRM, TME-Qualität), sondern vielmehr auf langzeitonkologischen Endpunkten basieren. Diese Ergebnisse stehen für die ALaCaRT-Studie noch aus.

\section{Korrespondenzadresse}

\section{J. Reibetanz}

Klinik für Allgemein-, Viszeral-, Gefäß- und

Kinderchirurgie,

Universitätsklinik Würzburg, 97080 Würzburg reibetanz_j@ukw.de

Interessenkonflikt. J. Reibetanz und C.T. Germer geben an, dass kein Interessenkonflikt besteht.

\section{Literatur}

1. Pas MH van der, Haglind E, Cuesta MA et al (2013) Laparoscopic versus open surgery for rectal cancer (COLOR II): short-term outcomes of a randomised, phase 3 trial. Lancet Oncol 14:210-218

2. Bonjer HJ, Deijen CL, Abis GA, Cuesta MA, van der Pas MH, de Lange-de Klerk ES, Lacy AM, Bemelman WA, Andersson J, Angenete E, Rosenberg J, Fuerst A, Haglind E, COLOR II Study Group (2015) A randomized trial of laparoscopic versus open surgery for rectal cancer. N Engl J Med 372(14):1324-1332 